\title{
A Simulation of Galaxy Formation by Radiation-SPH
}

\author{
Hajime Susa \& Masayuki Umemura \\ Center for Computational Physics, University of Tsukuba, Tsukuba 305, \\ Japan
}

\begin{abstract}
We investigate galaxy formation with Radiation-SPH simulations in order to find the effects of ultraviolet background radiation field on the star formation in the forming galaxies. We find the ultraviolet background radiation field can delay the star formation in protogalactic clouds, especially at low redshift $(z<2)$. This result suggests that galaxies formed at lower redshift tend to be late type galaxies, and early type galaxies tend to be formed at higher redshift.
\end{abstract}

\section{Introduction}

Formation of galaxies is a fundamental and a quite exciting issue in present-day cosmology. Recently, Susa \& Umemura (2000ab) propose that the self-shielding against UV background radiation regulates the star formation in pregalactic clouds. The star formation processes in primordial gas have been explored by many authors with $a b$ initio calculations (e.g. Matsuda, Sato \& Takeda 1969; Hutchins 1976; Carlberg 1981; Palla, Salpeter \& Stahler 1983; Susa, Uehara \& Nishi 1996; Uehara et al. 1996; Omukai \& Nishi 1998 ; Nishi et al. 1998; Nakamura \& Umemura 1999). The key physics is the radiative cooling by $\mathrm{H}_{2}$ line emission, because $\mathrm{H}_{2}$ is the only coolant for primordial gas at $T \lesssim 10^{4} \mathrm{~K}$. Susa \& Umemura (2000a) have studied the efficiency of $\mathrm{H}_{2}$ cooling in collapsing clouds exposed to UV background radiation, because it is significant after the reionization of the universe, probably at $z \lesssim 10$ (Nakamoto, Umemura, \& Susa 2001 , and references therein). Susa \& Umemura (2000a) have found that if a cloud undergoes the first sheet collapse at higher redshifts $(z \geq 4)$, then the cloud is quickly shielded against the UV background and consequently cools down due to the efficient formation of $\mathrm{H}_{2}$. Consequently, it leads to an early burst of star formation. On the other hand, the shielding is delayed for a later collapsing cloud at $z \lesssim 4$, resulting in the dissipational galaxy formation. As a result, the bifurcation branch of the self-shielding is corresponding to the boundary between the dissipationless and dissipational galaxy formation.

However, the theoretical approach employed in Susa \& Umemura (2000a) is so simplified ( $1 \mathrm{D}$ sheet collapse), that we have to investigate this bifurcation process in a more realistic situation.

In this paper, we perform numerical simulations of galaxy formation by Radiation-SPH code, developed by ourselves. In the next section, numerical methods are briefly summarized, and in section 3 , the parameters and initial 
conditions of numerical simulations are described. In section 4, results are explained, and the final section is devoted to the conclusions and discussions.

\section{Numerical Methods}

In this paper, we calculate the hydrodynamics by SPH, based on the formalism in Steinmetz \& Mülller (1993), except that we use the artificial viscosity by Monaghan (1992), and we use the SPH resize formalism in Thacker et al. (2001). The gravity is calculated by Barns-Hut tree code (Barns \& Hut 1986) provided by J. Makino.

Radiation transfer is the key physics of this calculation, and we use the method recently developed by Kessel-Dynet \& Burkert (2000). We also calculate the time dependent chemical reactions of primordial gas, in order to calculate radiative cooling by $\mathrm{H}_{2}$, which is the main coolant below $10^{4} \mathrm{~K}$ for $Z>10^{-2}$ gas. The code is already developed by Susa \& Kitayama (2000).

The basic test problems such as Sod's shock tube and Evrard's collapse are performed and the results agrees well with the analytic solution or the solution by $1 \mathrm{D}$ mesh code. We also performed the test of the propagation of ionization front. The results are shown in Fig.1. Incident ionizing flux comes from the left (negative $z$ ), and the ionized region propagates from the left to the right. Large open circles denote the result by the Radiation-SPH code, and small filled circles represent the solution calculated by the $1 \mathrm{D}$ mesh code. The two results agrees well with each other.

\section{Description of Runs}

Initial condition of the particle distribution is generated as follows: First, create the realization of density distribution of a cube using the package GRAFIC in COSMICS, assuming the CDM cosmology with $\Omega_{\mathrm{CDM}}=0.3, \Omega_{\Lambda}=0, h=0.7$. We put constraint on the realized density field, so that center of the cube is over-dense at a galactic scale. Then, we cut out a spherical region from the cube. We use this spherical particle distribution as the initial condition of the simulation. If we change the amount of over density at the center, we can adjust the formation epoch of the simulated galaxy. The total mass of the simulated individual galaxies are $2.6 \times 10^{10} M_{\odot}$, and both of the number of SPH particles and dark matter particles are 5000 .

An ionization source are located outside the simulated the galaxy, so that $I_{21}$ at the center of the simulated region to be as follows: $I_{21}=0.5[(1+z) / 3]^{3}$ for $z \leq 2$ and $I_{21}=0.5$ for $2<z \leq 4$. As for $z>4$ we employ the exponentially damping model, $I_{21}=0.5 \exp [3(4-z)]$ which is suggested by Umemura, Nakamoto \& Susa (2001).

"Star formation" is also taken into account, and this is quite different from the previous works. The conditions of star formation employed in our simulation are as follows: (1) $T_{\text {gas }} \leq 5000 \mathrm{~K}$ (2) fraction of $\mathrm{H}_{2}$ is larger than $5 \times 10^{-4}$ (3) $\rho \geq 200 \rho_{\text {univ }}$. These conditions ensures that the region is self-shielded against the external UV radiation field, so that the temperature can drop much below $10^{4} \mathrm{~K}$ by $\mathrm{H}_{2}$ cooling, which is expected for the star forming regions (Susa \& Kitayama 
2000). Here we emphasize that these conditions can never be employed in a calculation which do not include the effects of radiation transfer and the cooling mechanism below $10^{4} \mathrm{~K}$, such as $\mathrm{H}_{2}$ cooling. Once this condition is satisfied, the SPH particle is converted into star particle at the local free-fall time scale.

\section{Results}

In this section, we summarize the results obtained by the numerical calculations. We find two typical cases, whose results are qualitatively different from each other. Here we explain them in order.

\subsection{Formation of Galaxies at high redshift $(z \sim 4)$}

In this case, star formation proceeds from $z \sim 10$ to 3, and most of the gas of the galaxy is converted into star particles by $z \sim 3$. The distribution of resultant star particles are spherical (Fig.2). This result can be understood naturally, if we follow the paradigm of dissipationless galaxy formation. In this case, the local density perturbations collapse at high redshift $(z \gtrless 4)$, since the formation epoch of the galaxy is relatively high. Consequently, the locally collapsed gas clouds are not affected by the UV radiation field because of the weak background radiation intensity and high gas density of the universe. Thus, the collapsed gas can be converted star particles efficiently, and the collapse of the whole galaxy proceeds in a dissipationless fashion.

\subsection{Formation of Galaxies at lower redshift $(z \sim 2)$}

In this case, star formation starts at $z \sim 5$ and the starburst phase continues until $z \sim 3$. After the burst phase, star formation proceeds moderately, until present. In this slow star forming phase, the surrounding gas accretes to the central star cluster, as if it is forming a bulge-disc system. In Fig.3, the particle distributions at $z \sim 0.5$ is presented. As you can see, rotationally supported gas disk is formed, and the distribution of star particles are more flattened than the previous case. Actually, the rotation of the stellar system is more important than the previous case (rotation energy of the stellar system is comparable to the energy of random motion). Obviously, this case corresponds to the dissipative galaxy formation: collapse at lower redshift inhibits the fast star formation even in a collapsing region, because of the UV. penetration. Thus, the gas collapses isothermally keeping $T_{\text {gas }} \sim 10^{4} \mathrm{~K}$, and loses significant amount of the thermal energy before the star formation is triggered.

\section{Conclusion \& Discussion}

Here we studied galaxy formation with Radiation-SPH simulations in order to find the effects of ultraviolet background radiation field on the star formation in the forming galaxies. We found the ultraviolet background radiation field can delay the star formation in protogalactic clouds, especially at low redshift $(z<2)$. The resultant galaxies formed at lower redshift tend to have late type morphology, and early type galaxies tend to be formed at higher redshift. 
However, number of problems still remain: (1) The mass of the galaxy is too small, (2) Effects of SN is not included, (3) Metal pollution is not taken into account. Among these problems, (2) might reduce the amount of stars formed in bulge system, because of the galactic wind. On the other hand, (3) might enhance the star formation efficiency, due to the metal cooling below $10^{4} \mathrm{~K}$ and the UV shielding by dust. These two are very important, but will not change the qualitative results of the morphological bifurcation.

On the contrary, problem (1) is very serious, because we are simulating only dwarf galaxies. The mass of the galaxy are actually limited by the numerical resolution. If we try to resolve the self-shielded region, we have to resolve $M_{\text {gas }} \sim$ $10^{6} M_{\odot}$ (Susa \& Kitayama 2000). Thus, the number of SPH particles directly determine the total mass of the simulated galaxy. From this point of view, we are now planning to enhance this simulation to a larger one. In order to enhance these simulations, we have to accelerate two major parts of the code: first one is the calculation of radiation transfer + chemical reactions and the second is the gravitational force calculation. The former can be accelerated simply by the parallelization on massively parallel supercomputer. The latter is, however, cannot be accelerated directly, because tree codes are incompatible with massively parallel computer system. Thus, we might need special purpose computer (e.g. GRAPE) for the calculation of gravitational force. Moreover, the gravity calculator needs to communicate with another supercomputer system which can deal with the radiation transfer and chemical reactions. If such a system is completed, it is expected to enable us to simulate the galaxies which is 1-2 orders of magnitude larger than the present calculation.

Acknowledgments. We thank Jun Makino, who provided the tree code. The analysis of this paper has been made with computational facilities at the Center for Computational Physics in University of Tsukuba. This work is supported in part by Research Grant of University of Tsukuba Research Project (HS), and the Grant from Japan Society for the Promotion of Science (HS 13740124; MU 11640225). 


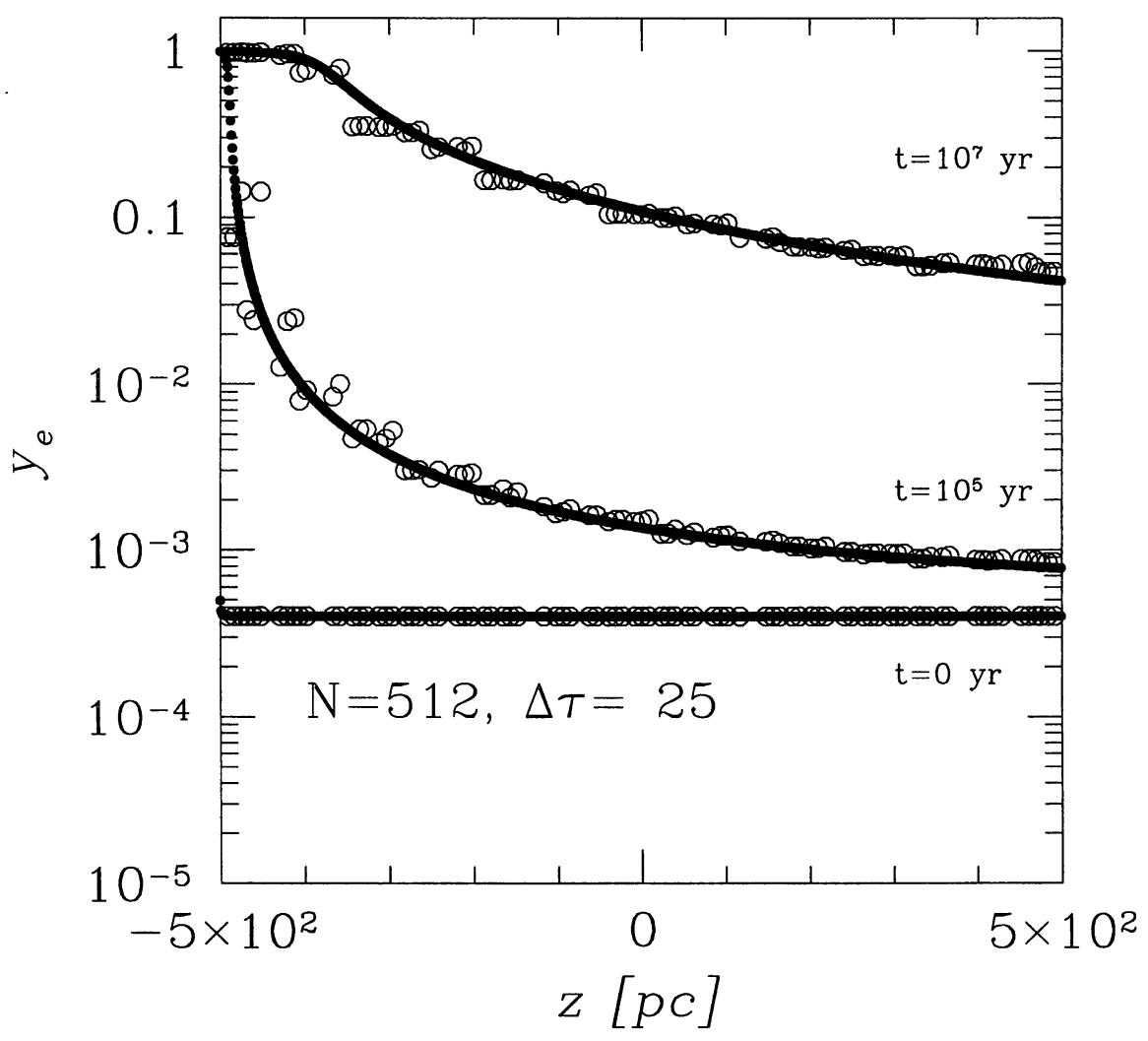

Figure 1. Evolution of spatial distribution of ionization degree in a uniform cube is presented. The vertical and horizontal axes denote the ionization degree of the gas, and the spatial coordinate which is parallel to the ray of the incident photons, respectively . The incident ionization flux comes from the left (negative $z$ ) with $I_{\nu} \propto \nu^{-1}$. Large open circles denote the result of numerical calculation by RadiationSPH code, and the small filled circles denote the result of $1 \mathrm{D}$ mesh code. Three sets of circles represent three snapshots for three different time steps. 

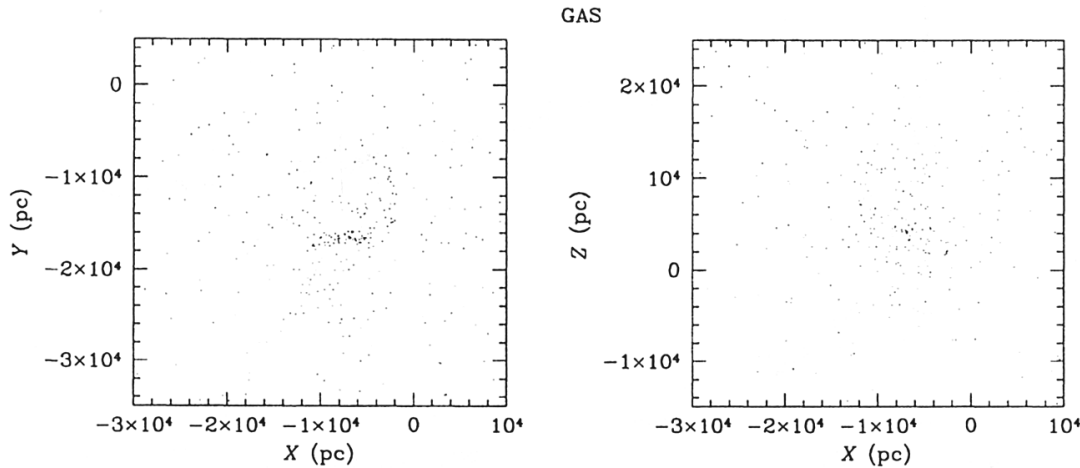

STARS
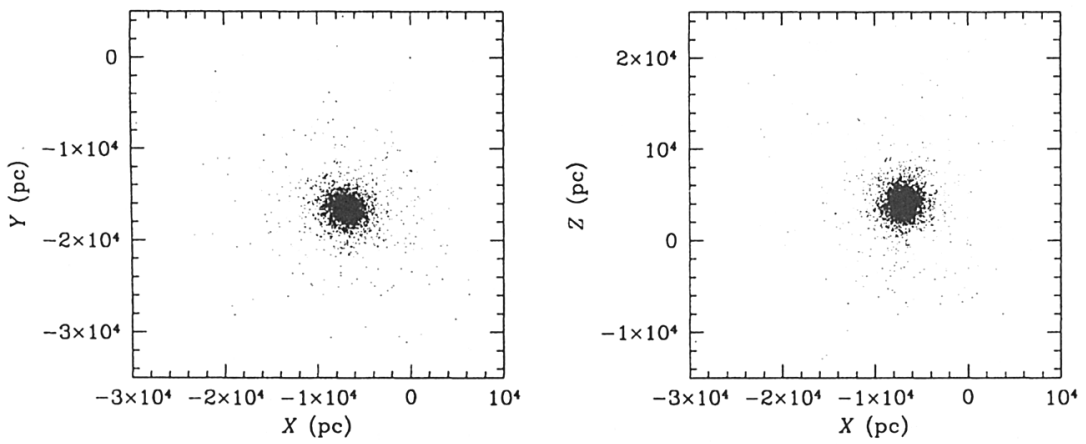

Figure 2. A galaxy with collapse redshift $z_{\mathrm{c}} \sim 4$ is presented. The figure is the snapshot at $z=0.5$ The upper two panels show the location of SPH particles on X-Y and X-Z plane. The two panels at the bottom show the numerically formed star particles. 

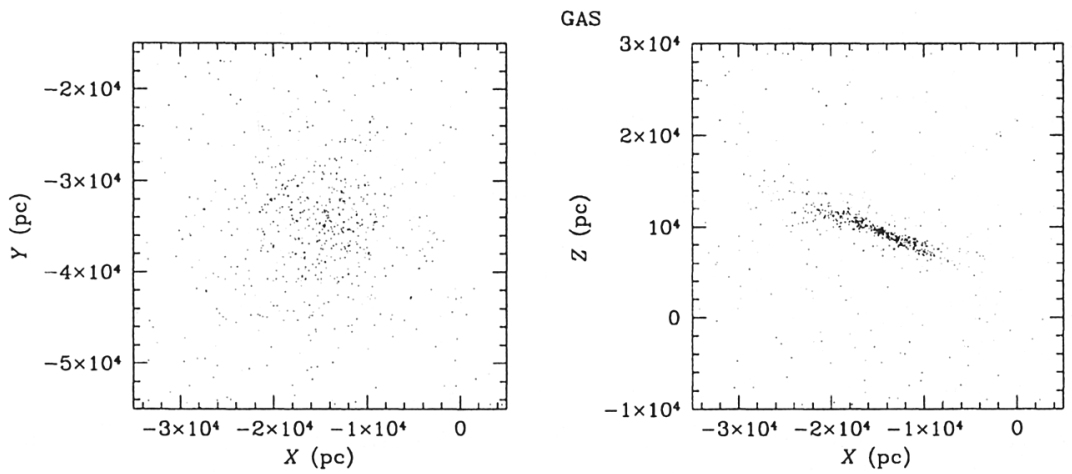

STARS
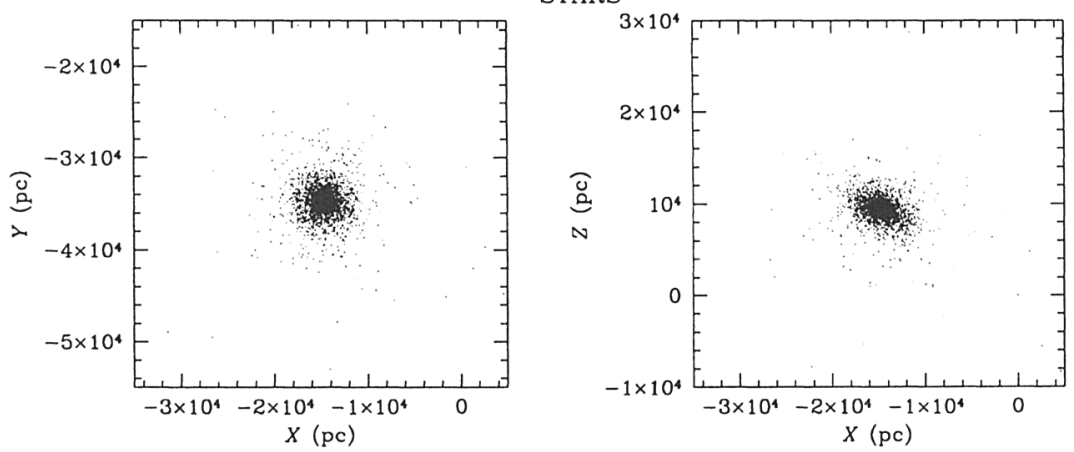

Figure 3. Same as Fig.2 except that the formation epoch is $z \sim 2$. 


\section{References}

Barns, J. \& Hut, P. 1986, Nature, 324, 446

Carlberg, R. G. 1981, MNRAS, 197, 1021

Hutchins, J. B. 1976, ApJ, 205, 103

Kessel-Dynet, O. \& Burkert, A. 2000, MNRAS, 315, 713

Matsuda, T., Sato, H., \& Takeda, H. 1969, Prog. Theor. Phys. 42, 219

Monaghan, J.J. 1992, ARA\&A, 30, 543

Nakamoto, T., Umemura, M., \& Susa, H. 2001, MNRAS, 321, 593

Nakamura, F., \& Umemura, M. 1999, ApJ, 515, 239

Nishi, R., Susa, H., Uehara, H., Yamada, M. \& Omukai, K. 1998, Prog. Theor. Phys., 100, 881

Omukai, K., \& Nishi, R. 1998, ApJ, 508, 141

Palla, F., Salpeter, E. E., \& Stahler, S. W. 1983 ApJ, 271, 632

Steinmetz, M. \& Müller, E. 1993, å, 268, 391

Susa, H. \& Kitayama, T. 2000, MNRAS, 317, 175

Susa, H., Uehara, H., \& Nishi, R. 1996, Prog. Theor. Phys., 96, 1073

Susa, H., \& Umemura, M. 2000a, ApJ, 537, 578

Susa, H., \& Umemura, M. 2000b, MNRAS, 316, L17

Thacker, R. J., Tittley, E. R., Pearce, F. R., Couchman, H. M. P., Thomas, P. A. 2000, MNRAS, 319, 619

Uehara, H., Susa, H., Nishi, R., Yamada, M., \& Nakamura, T. 1996, ApJ, 473, L95

Umemura, M., Nakamoto, T., \& Susa, H. 2001, in preparation 